\title{
Genome- and Transcriptome-Wide Characterization of bZIP Gene Family Identifies Potential Members Involved in Abiotic Stress Response and Anthocyanin Biosynthesis in Radish (Raphanus sativus L.)
}

\author{
Lianxue Fan, Liang Xu, Yan Wang, Mingjia Tang and Liwang Liu *(1) \\ National Key Laboratory of Crop Genetics and Germplasm Enhancement, Key Laboratory of Horticultural Crop \\ Biology and Genetic Improvement (East China) of MOAR, College of Horticulture, Nanjing Agricultural \\ University, Nanjing 210095, China; 2016204023@njau.edu.cn (L.F.); nauxuliang@njau.edu.cn (L.X.); \\ wangyanhs@njau.edu.cn (Y.W.); 2017204022@njau.edu.cn (M.T.) \\ * Correspondence: nauliulw@njau.edu.cn
}

Received: 30 October 2019; Accepted: 13 December 2019; Published: 16 December 2019

\begin{abstract}
Basic leucine zipper (bZIP) transcription factors play crucial roles in various abiotic stress responses as well as anthocyanin accumulation. Anthocyanins are most abundant in colorful skin radish, which exhibit strong antioxidant activity that offers benefits for human health. Here, a total of 135 bZIP-encoding genes were identified from radish genome. Synteny analysis showed that 104 radish and 63 Arabidopsis bZIP genes were orthologous. Transcriptome analysis revealed that 10 RsbZIP genes exhibited high-expression levels in radish taproot (RPKM $>10)$. Specifically, RsbZIP010 exhibited down-regulated expression under $\mathrm{Cd}, \mathrm{Cr}$ and Pb stresses, whereas RsbZIP031 and RsbZIP059 showed significant down-regulation under heat and salt stresses, respectively. RT-qPCR analysis indicated that RsbZIP011 and RsbZIP102 were significantly up-regulated in the tissues of radish with high anthocyanin contents. Furthermore, the promoter sequences of 39 anthocyanin-related genes were found to contain G-box or ACE-box elements that could be recognized by bZIP family members. Taken together, several RsbZIPs might be served as critical regulators in radish taproot under $\mathrm{Cd}, \mathrm{Cr}, \mathrm{Pb}$, heat and salt stresses. RsbZIP011 and RsbZIP102 were the potential participants in anthocyanin biosynthesis pathway of radish. These results facilitate further investigation on functional characterization of $b$ ZIP genes in response to abiotic stress and anthocyanin biosynthesis in radish.
\end{abstract}

Keywords: bZIP gene; Raphanus satious L.; abiotic stress; anthocyanin biosynthesis; gene expression

\section{Introduction}

The basic leucine zipper (bZIP) family, as one of the largest and most diverse transcription factor (TF) families, is an important participator in various biological processes, such as seed maturation and germination, biotic or abiotic stress responses and hormone signaling [1,2]. It is widely known that bZIP TF possesses a conserved bZIP domain composed of two structural features, a DNA-binding basic region and a dimerizing leucine (Leu) zipper region [1,3]. The basic region of approximately 16 amino acids (aa) is highly conserved containing a nuclear localization signal followed by an invariant $\mathrm{N}-\mathrm{X}_{7}-\mathrm{R} / \mathrm{K}$ motif that binds to specific DNA sequences with an ACGT core, such as A-box (TACGTA), C-box (GACGTC) and G-box (CACGTG) [4]. The Leu zipper has a less conserved dimerization motif and is composed of a heptad repeat of Leu or other similar hydrophobic aa (e.g., Ile, Met, Phe or Val) positioned exactly nine aa towards the C-terminus, creating an amphipathic helix $[5,6]$. To bind with 
DNA, two subunits adhere via interactions between the hydrophobic sides of their helices, which creates a superimposing coiled-coil structure for recognition and dimerization [2,5].

The bZIP TF family has been extensively identified in various plant species, such as Arabidopsis, rice, rapeseed, tomato and apple [1,7-10]. The Arabidopsis bZIP gene family consisted of 78 members are assorted into 13 groups (Groups A-K, M and S) [1]. Recently, several bZIPs were found to be involved in response to a wide range of abiotic stresses, such as heat and high-salinity stresses [11,12]. Interestingly, increasing evidences indicate that a few bZIP genes such as HY5 (Elongated hypocotyl 5) and $H Y H$ (HY5 homolog) are involved in the biosynthesis pathway of anthocyanins in plants [13-15]. In Arabidopsis, HY5 can directly bind the promoters of some MYB TF genes, or together with other MYB factors, induce the expression of biosynthetic enzyme genes associated with PHYTOCHROME INTERACTING FACTOR3 activity for the promotion of anthocyanin accumulation [14]. Similarly, HY5 has been found to play a vital role in anthocyanin biosynthesis in tomato and apple $[13,15]$. These results provide useful information for comprehensive identification and characterization of $b$ ZIPs in root vegetable crops.

Radish (Raphanus sativus L.), belonging to the Brassicaceae family, is a major root vegetable crop with plenty of nutrition, and its taproot is an edible and stable food supplying a great source of phytochemicals such as anthocyanins $[16,17]$. Owning to the rapidly changeable climate and environment, radish growth and production are often severely limited by various abiotic stresses such as high temperature, salinity or metal pollution [18-23]. Although some bZIP TFs have been proven to be associated with different abiotic stress responses in many plant species, little information on the characterization of bZIP family is available in radish. The first draft sequences of the radish genome were released into the Raphanus sativus Genome DataBase (http://radish.kazusa.or.jp/) in 2014 [16], and its available genomic sequences and proteins/mRNA transcriptomics data provided valuable information for systematic identification of functional genes and TFs associated with important horticultural traits in radish. In this study, to identify the candidate genes that may participate in abiotic stress response and anthocyanin biosynthesis in radish, the $b Z I P$ gene family members were systematically identified at the genome-wide level. Herein, a total of $135 \mathrm{bZIP}$ gene members were identified based on the radish genome sequences, and the chromosome distribution, gene structure and conserved motifs were further investigated. Moreover, the expression patterns of RsbZIP genes were analyzed in different tissues, and seven RsbZIP genes that exhibited specific expression with high-levels in taproot were explored as the possible regulators in response to various stresses conditions such as cadmium $(\mathrm{Cd})$, chromium $(\mathrm{Cr})$, lead $(\mathrm{Pb})$, heat and salt stresses. Furthermore, two potential bZIP genes related to anthocyanin biosynthesis were firstly identified in radish. These results could provide valuable information on evolutionary relationship and functional divergence of the $b$ ZIP gene family in radish.

\section{Results}

\subsection{Identification and Chromosome Distribution of bZIP Family in Radish}

Using the HMM profile analysis, a total of 137 protein sequences were identified containing bZIP domains from the radish genome. Further SMART and InterPro analyses revealed that 135 genes could be assigned as radish $b Z I P$ genes, which were named RsbZIP001 RsbZIP135 accordingly (Table S1). Among them, 128 of 135 RsbZIP members were localized on the nine chromosomes (Chrs) of radish (Figure 1a,b). To be specific, Chr6 contained the highest number of RsbZIP genes (31, 23\%), followed by $\operatorname{Chr} 4(25,19 \%)$, while minimum genes were distributed on Chr1 $(6,4 \%)$ (Figure 1a). The tandem and segmental duplication events were also investigated. In all, only three pairs of tandem duplicates in RsbZIP gene family were determined, including RsbZIP005-RsbZIP006, RsbZIP037-RsbZIP038 and RsbZIP085-RsbZIP086 (Figure 1b). Moreover, 27 (20\%) segmental duplicated RsbZIP genes were detected to localize on two different chromosomes (Table S2). 
(a)

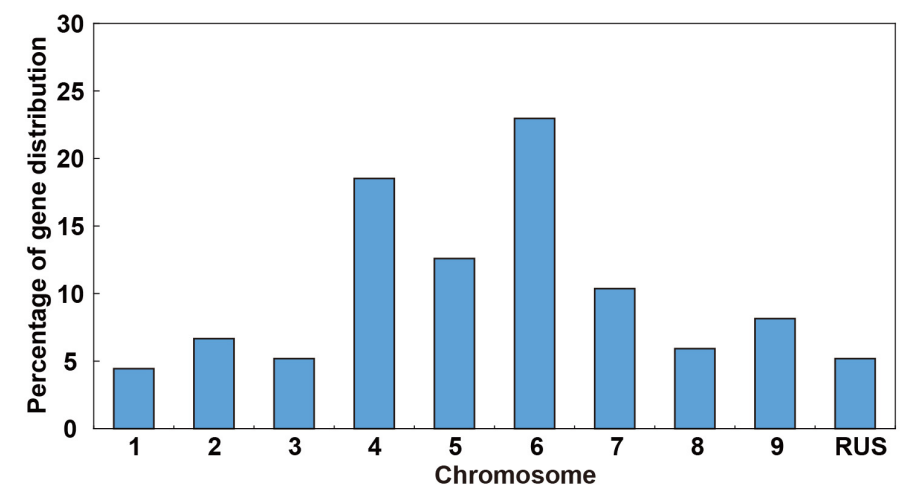

(b)

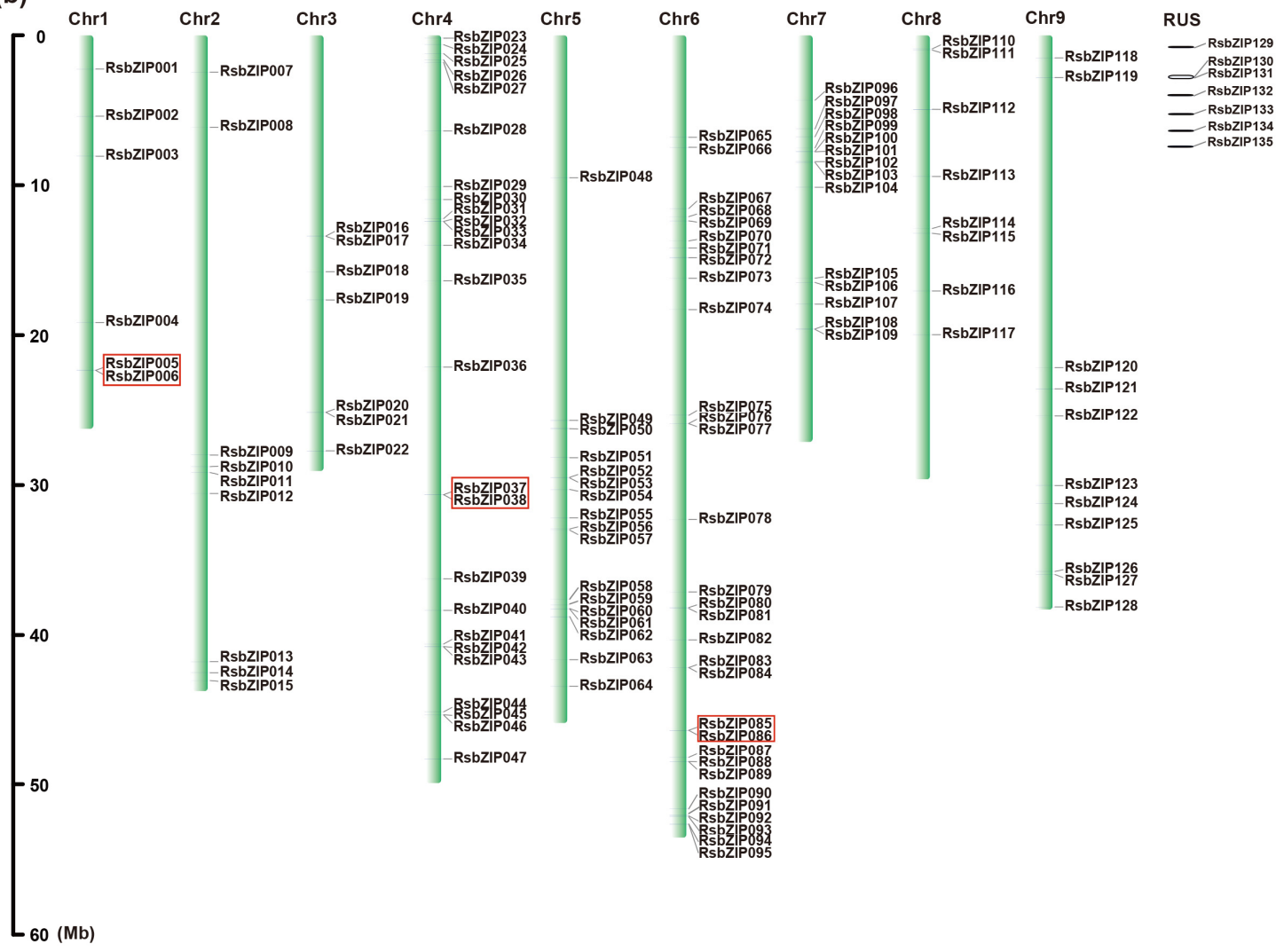

Figure 1. Chromosome localization and distribution of duplication events of radish $b Z I P$ genes. (a) Percentage of $b Z I P$ genes on each radish chromosome. (b) The RsbZIP genes were localized onto radish chromosomes (Chr1-9). Tandem duplicated genes on a particular chromosome are indicated in the box. RUS represents radish unassigned scaffolds.

\subsection{Gene Structure and Conserved Motif Analysis of RsbZIPs}

Exon-intron organizations of the 135 RsbZIPs were investigated to obtain the gene structure and evolutionary trajectory. The number of introns varied from 0 to 12 in RsbZIP genes and the genes that clustered together generally possessed similar gene structures (Figure 2a,b). A total of $37(27 \%)$ RsbZIPs with no intron were detected, including 35 from Group $\mathrm{S}$ and two from Group A. Groups $\mathrm{B}, \mathrm{C}, \mathrm{E}, \mathrm{F}, \mathrm{H}, \mathrm{J}$ and $\mathrm{M}$ had 1-6 introns, whereas the RsbZIP genes containing a minimum of 7 introns appeared in Groups D, G and I (Figure 2a,b).

A total of 20 conserved motifs were identified from all the RsbZIP proteins. Interestingly, the RsbZIPs within the same group exhibit similar motif compositions, which further supported the group classification. Motif 1 was the basic region and the hinge of bZIP domain, which existed in all 135 RsbZIP proteins. Motifs 2, 5, 8 and 10 represented the variable motifs in the Leu zipper region 
across the radish bZIP family (Figure 2c, Figure S1). For example, Motif 5 appeared in Groups I and E, and Motif 8 appeared in Group D, while the Motif 10 only appeared in Groups S and C (Figure 2a,c). The number of Leu units ranged from 3 to 7, and some of them were interrupted by one or two other units which are conserved with the same number of aa such as RsbZIP044 (Figure S1). Several additional motifs were also identified in some groups. For instance, most members from Group A shared Motifs 9, 11, 12, 18 and 20, while Group B possessed Motifs 3, 16 and 19. The members from Group D harbored Motifs 3, 4, 6 and 7, while Group G had Motifs 15 and 16, and Group I shared Motifs 14 and 17. The Group E possessed Motif 13 exclusively, whereas Groups C, F, H, J and M lacked any additional motif (Figure 2a,c). These specific motifs might imply diverse functions of the bZIP family in radish.

(a)

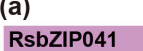

RsbZIP093

RsbZIP049

RsbZIP111

RsbZIP023

RsbZIP044

RsbZIP037

RsbZIP001

RsbZIP110

RsbZIP039

RsbZIP086

RsbZIP072

RsbZIP056

RsbZIP057

RsbZIP084

RsbZIP038

RsbZIP083

RsbZIP103

RsbZIP067

RsbZIP126

RsbZIP016

RsbZIP017

RsbZIP090

RsbZIP047

RsbZIP054

RsbZIP108

RsbZIP109

RsbZIP099 M

RsbZIP042

RsbZIP092

RsbZIP130

RsbZIP028

RsbZIP102

RsbZIP063

RsbZIP065

RsbZIP082

RsbZIP011

RsbZIP013

RsbZIP058

RsbZIP045

RsbZIP046

RsbZIP022

RsbZIP050
RsbZIP135

RsbZIP135

RsbZIP101

RsbZIP101
RsbZIP064

RsbZIP009

RsbZIP119

RsbZIP106

RsbZIP118

RsbZIP112

RsbZIP112

RsbZIP098

RsbZIP128

RsbZIP062

RsbZIP069

(b)
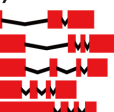

NM
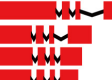

ac

MHMW-WMM
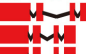

r

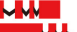

MM

(19u

WV
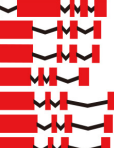

MN

IMM
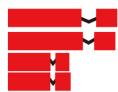

MMN

Mn

iv

vin

Mrin

MV

MM

$\mathrm{M}$

MN

MM

M-1

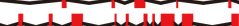

MWATIM

MWM 14

M.M

Wh

chin

Mit 4

9.7.

Tur

Whiv

atr

witiv

사는

5'

\begin{tabular}{llllllllll}
\hline 0 & 1 & 2 & 3 & 4 & 5 & 6 & 7 & 8 & 9
\end{tabular}

(c)
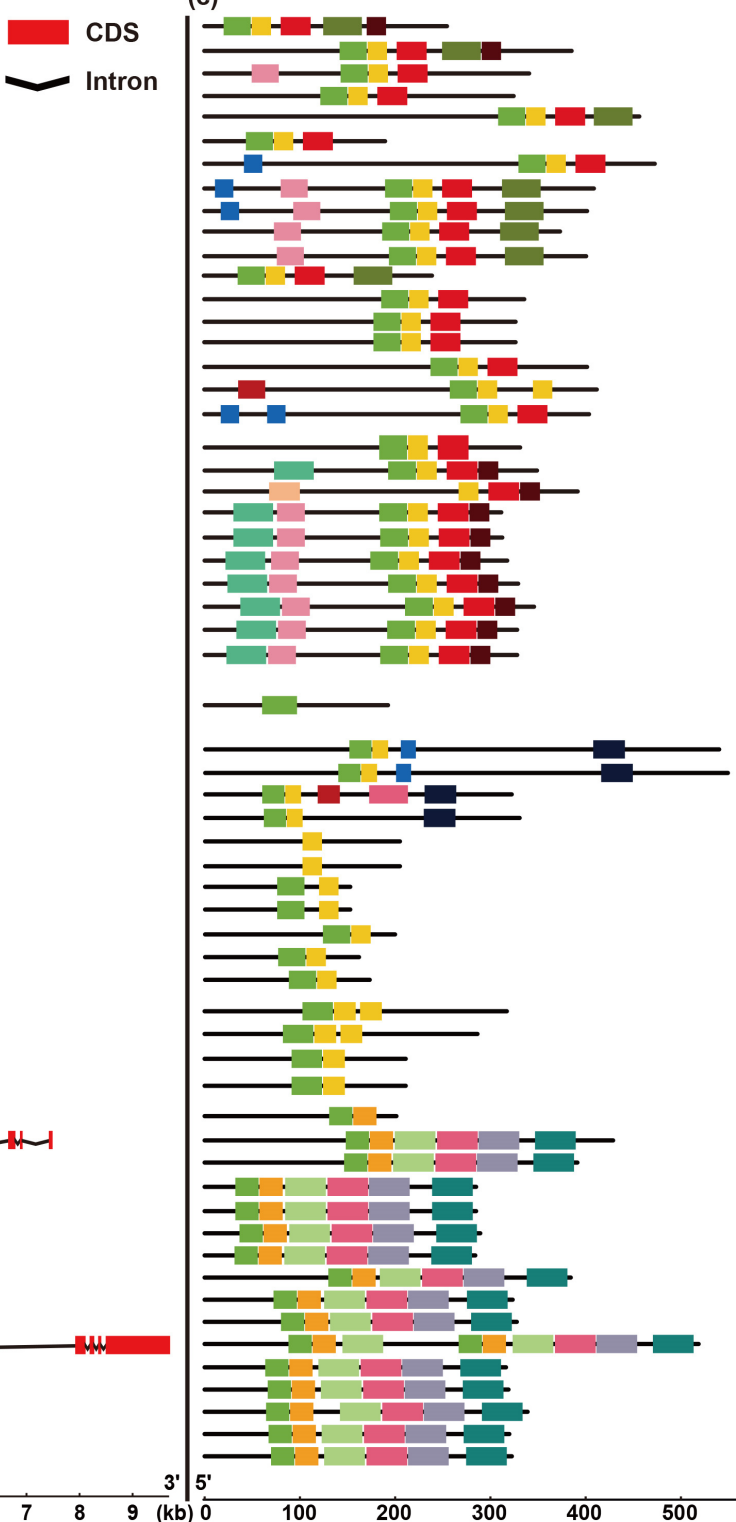

(kb) 0

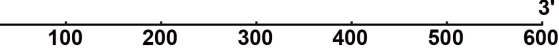

Figure 2. Cont. 
(a) RsbZIP076 RsbZIP077 RsbZIP025 RsbZIP036 RsbZIP116

RsbZIP080

RsbZIP080

RsbZIP052

RsbZIP05

RsbZIP131

RsbZIP131

RsbZIP043

RsbZIP091

RsbZIP088

RsbZIP089

RsbZIP040

RsbZIP004

RsbZIP005

RsbZIP006

RsbZIP027

RsbZIP115

RsbZIP066

RsbZIP048

RsbZIP133

RsbZIP029

RsbZIP070

RsbZIP124

RsbZIP060

RsbZIP061

RsbZIP068

RsbZIP079

RsbZIP127

RsbZIP015

RsbZIP024

RsbZIP032

RsbZIP033

RsbZIP123

Rs?IP055

(10)

(

RsbZIP104

RsbZZP007

RsbZIP105

RsbZIP026

RsbZIP114

RsbZIP075

RsbZIP059

RsbZIP122

RsbZIP134

RsbZIP035

Rsb7IP129

(

(2)

RsbZIP125

RsbZIP002

RsbZIP078

RsbZiP008

RsbZIP034

RsbZIP073

RsbZIP012

RsbZIP096

RsbZIP020

RsbZIP021

RsbZIP030

RsbZIP071

RsbZIP031

RsbZIP120

RsbZIP018
RsbZIP051

RsbZIP003

RsbZIP019

RsbZIP087

RsbZIP117

RsbZIP014

RsbZIP113

RsbZIP121

RsbZIP094

RsbZIP095

(b)

$\mathrm{MH}$

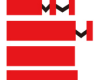

-

vi

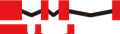

M

$\mathrm{MM}^{\mathrm{M}}$

MM

$+$

MM

(1)

(n)

-

$\square$

r

$-$

$+$

$\square$

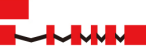

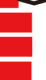

5

$\square$

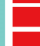

5

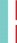

E

$E$

$\square$

E-

$\square$

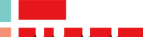

पnu

- N

un

un

CUM

MNWWWVI

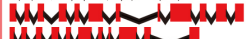

MVMUN

WVIVWNVI

MVNWNW

WVW WVV

WNW WVI

$\mathbf{5}^{\prime}$

$3^{3} 5$

(c)

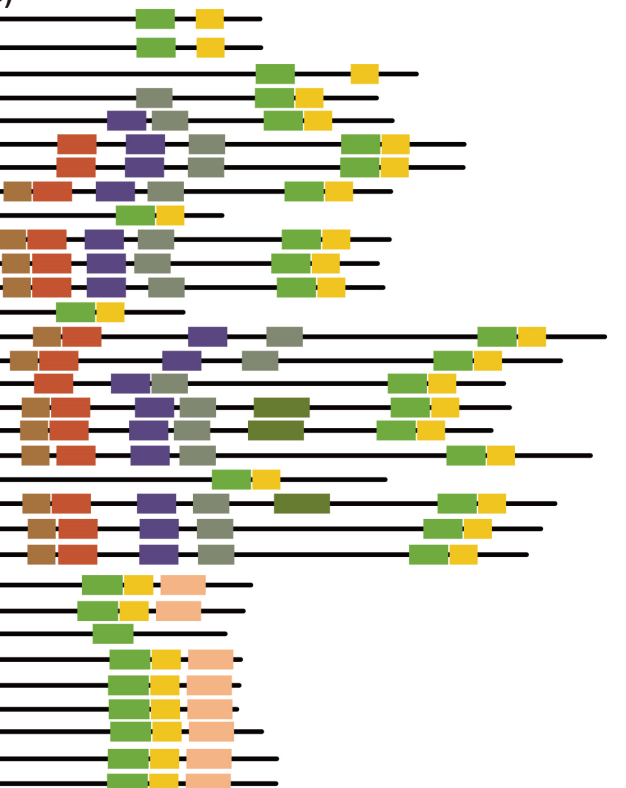

Figure 2. Characterization of radish $b Z I P$ genes. (a) The clustering of RsbZIP proteins based on Neighbor-Joining phylogenetic tree. (b) Exon-intron structures of RsbZIP genes. Red box and black line indicate exon and intron, respectively. (c) Distribution of conserved motifs for RsbZIP proteins.

\subsection{Phylogenetic and Evolutionary of Radish and Arabidopsis bZIP Genes}

To investigate the phylogenetic relationship of the $b Z I P$ gene families, the aa sequences encoded by the $b Z I P$ genes from radish and Arabidopsis were used to construct an unrooted Neighbor-Joining (NJ) tree (Figure 3, Table S1). As shown in Figure 3, the 213 bZIP proteins could be divided into 13 
subfamilies, which were named as Groups A, B, C, D, E, F, G, H, I, J, K, M and S according to the Arabidopsis classification [1]. Group $\mathrm{S}$ was the largest subfamily, consisting of 37 RsbZIP members, followed by the Groups A and I containing 23 and 18 RsbZIP proteins, respectively. The 16 and 10 RsbZIP members were classified into Groups D and E, while the number of members in Groups B, H, F, C and G was four, seven, four, six and eight, respectively. Both Groups J and M only had one RsbZIP protein, respectively. Interestingly, the Group K included only AtbZIP60 without any RsbZIP member, indicating that this group might be specific to Arabidopsis (Figure 3). According to the functional and regulatory aspects of the bZIP subgroups in Arabidopsis, the classification of RsbZIP gene family might serve as their phylogeny-based functional prediction.

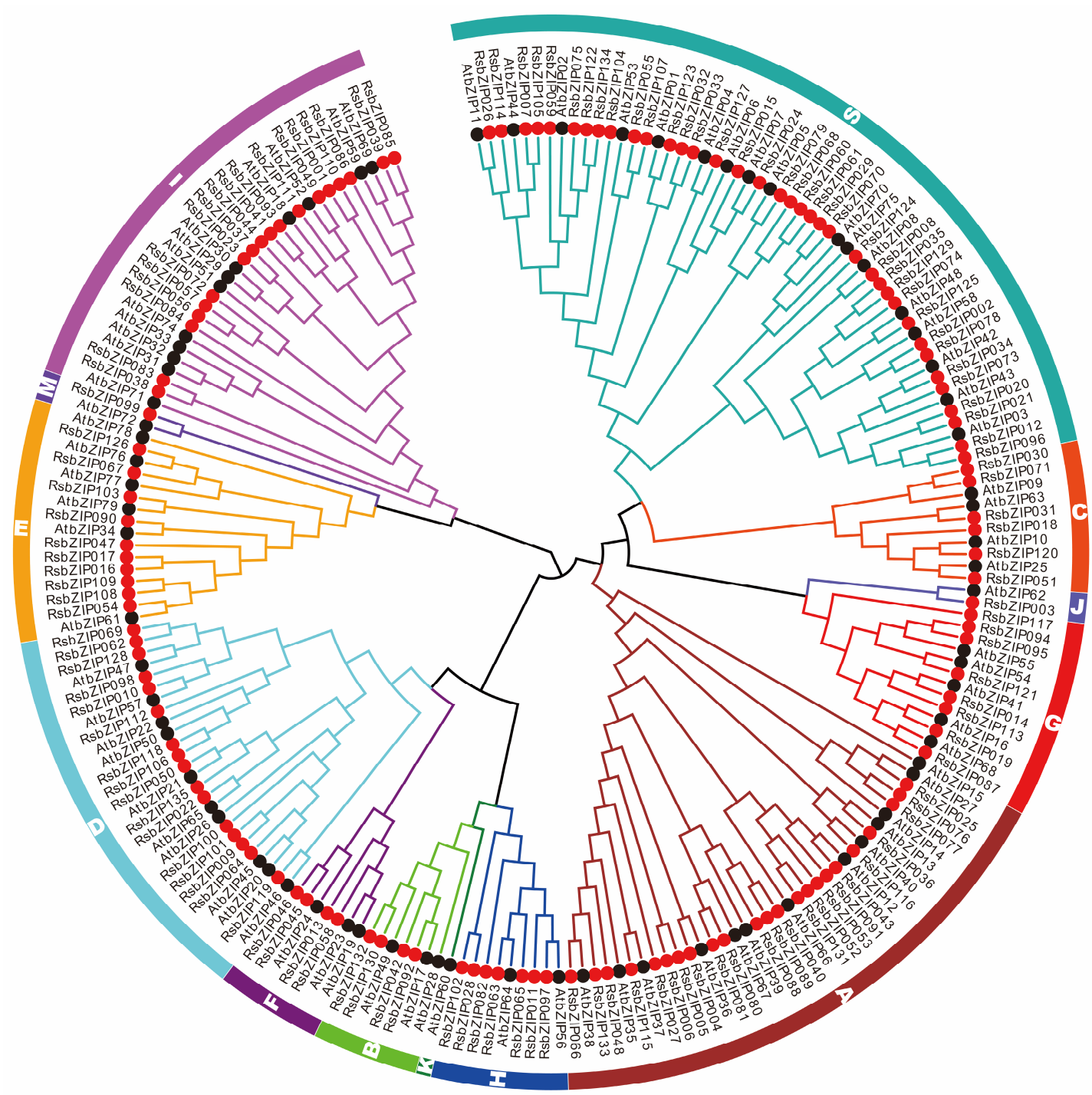

Figure 3. Phylogenetic tree of bZIP proteins from radish (RsbZIP) and Arabidopsis (AtbZIP). The colored branches and outer races indicate different subgroups, and the red solid and black circles indicate RsbZIP and AtbZIP proteins, respectively.

To deduce the origins and evolutionary history of $b$ ZIP genes, a comparative bZIP synteny map was conducted between radish and Arabidopsis. Large-scale syntenies showed that 104 radish and 63 Arabidopsis bZIP genes were orthologous (Figure 4, Table S3). Among them, 12 pairs of syntenic orthologous genes (one to one) were identified, such as RsbZIP003-AtbZIP62, RsbZIP050-AtbZIP21 and RsbZIP056-AtbZIP51. These genes may be derived from a common ancestor of radish and Arabidopsis. Two syntenic orthologous gene pairs with one radish gene corresponding to multiple Arabidopsis genes 
were also identified including RsbZIP087-AtbZIP16/68 and RsbZIP094-AtbZIP54/55. Correspondingly, there also existed syntenic orthologous gene pairs with one Arabidopsis gene corresponding to multiple radish genes, AtbZIP01-RsbZIP032/123, AtbZIP09-RsbZIP030/071 and AtbZIP41-RsbZIP014/113. Additionally, the gene pairs where two Arabidopsis genes correspond to the same (two, three or four) radish genes were also found, such as AtbZIP13/40-RsbZIP036/116, AtbZIP22/50-RsbZIP106/112/118 and AtbZIP59/69-RsbZIP001/039/085/110 (Figure 4, Table S3). A range of synteny events suggested that many $b Z I P$ genes arose before the divergence of the Arabidopsis and radish lineages.

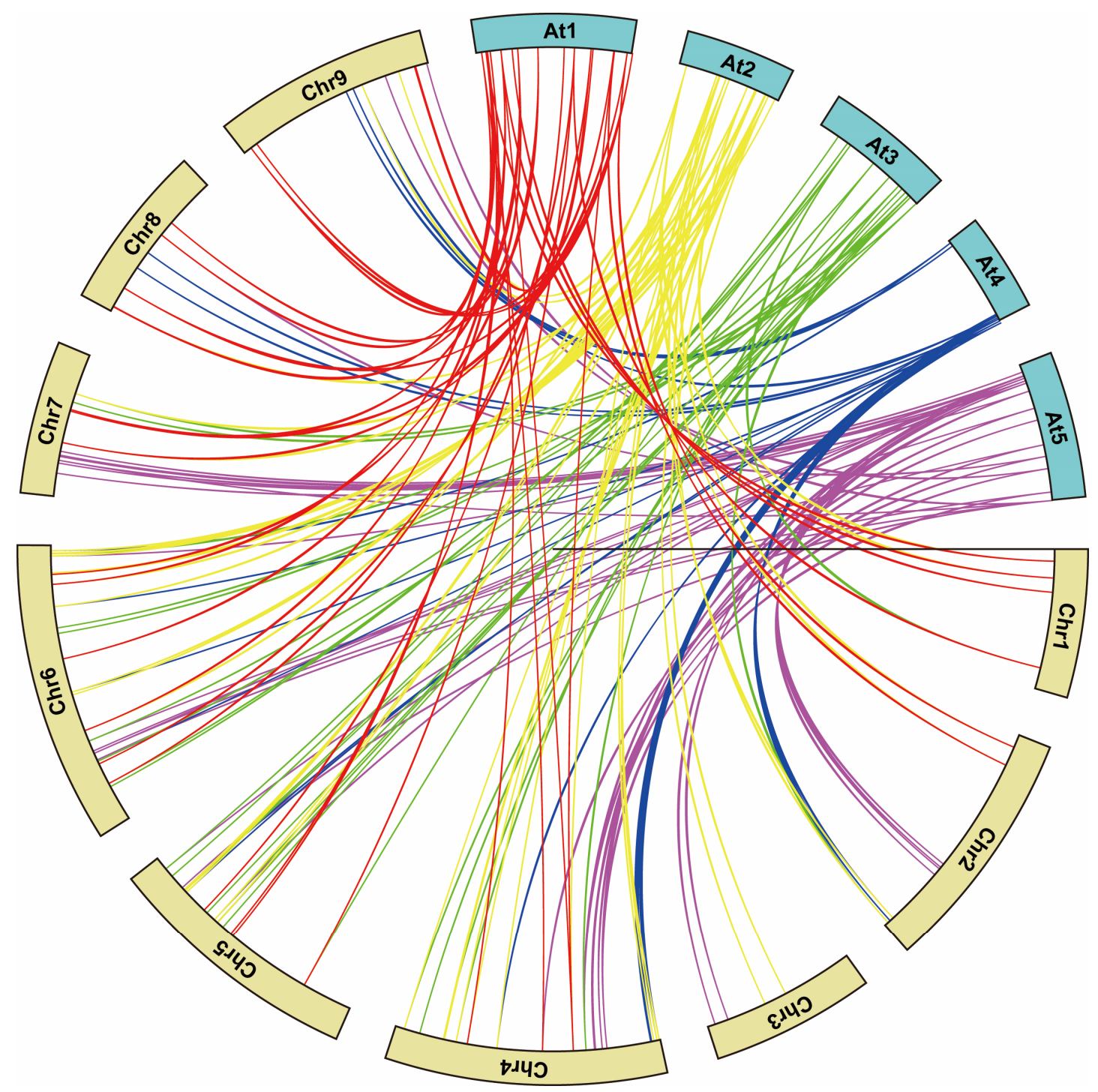

Figure 4. Synteny analysis of bZIP genes between radish and Arabidopsis. Colored lines connecting two chromosomal regions indicate syntenic regions between radish (Chr1-9) and Arabidopsis (At1-5) chromosomes.

\subsection{Expression Profiles of bZIP Genes in Different Tissues of Radish}

To investigate the tissue-specific expression profiles of $b Z I P$ genes in different developmental stages of radish, an expression analysis of RsbZIP genes in five tissues (leaf, cortical, cambium, xylem and root tip) were displayed with RNA-seq data from Mitsui et al. [18]. A heatmap was conducted based on the reads per kilobase per million reads (RPKM) values of 135 RsbZIP genes (Figure 5a). All RsbZIP genes exhibited very diverse expression profiles in five tissues. On average, the expression of $68 \%, 61 \%, 57 \%, 51 \%$ and $47 \%$ RsbZIP genes were detected in these five different tissues, when 
RPKM values were greater than 2, 4, 6, 8 and 10, respectively (Figure 5b, Figure S2). In detail, 39\%, $47 \%, 45 \%, 50 \%$ and $51 \%$ RsbZIP genes showed higher transcriptional abundance (RPKM value $>10$ ) in leaf, cortical, cambium, xylem and root tip, respectively (Figure 5b). Among them, 41 RsbZIP genes including RsbZIP001/004/009 were abundantly expressed (RPKM value $>10$ ) in all tissues (Figure 5c,d), while 31 genes (e.g., RsbZIP007/008/016) were expressed at relatively low levels. Moreover, a total of 10 RsbZIPs (e.g., RsbZIP031/059/064) were selectively expressed with high-levels (RPKM value>10) in taproot (cortical, cambium, xylem and root tip), while four RsbZIPs (e.g., RsbZIP047/063/069) were only in leaf with high-level expression (Figure 5a). These RsbZIP genes might play important roles in radish growth and development process.

(a)
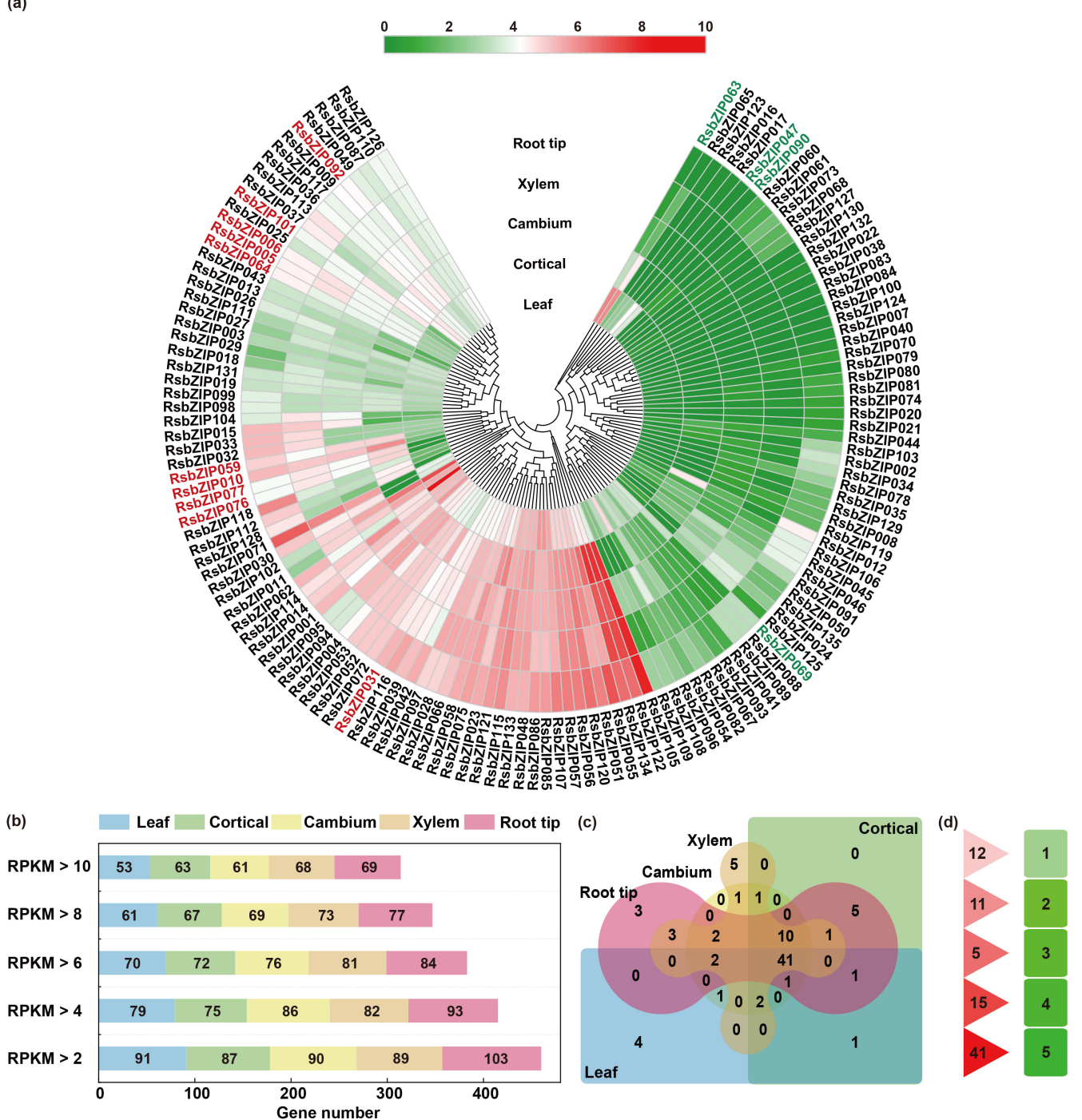

Figure 5. Expression profiles of RsbZIP genes in different tissues of radish. (a) Heatmap of expression levels for RsbZIP genes in five tissues (leaf, cortical, cambium, xylem and root tip). The heatmap was constructed based on the RPKM values of RsbZIPs. Changes in gene expression are shown in color as the scale. Red and green fonts indicate the selectively expressed genes with high-levels (RPKM>10) in taproot (cortical, cambium, xylem and root tip) and leaf, respectively. (b) The number of RsbZIP genes with transcriptional abundance $(R P K M>10)$ in each tissue. (c) Venn diagram of overlapping RsbZIPs that are abundantly expressed in different tissues. (d) Number of RsbZIP genes that exhibited abundant expression in 1, 2, 3, 4 and 5 tissues. 


\subsection{Potential Roles of RsbZIP Genes in Various Abiotic Stress Responses}

The expression patterns of $\mathrm{Rs} b \mathrm{ZIP}$ genes under $\mathrm{Cd}, \mathrm{Cr}, \mathrm{Pb}$, heat and salt stresses were investigated in radish according to previously root transcriptome data [19-23]. As shown in Figure 6a, a total of 76, 77, 38, 43 and 46 RsbZIP genes were differentially expressed under $\mathrm{Cd}, \mathrm{Cr}, \mathrm{Pb}$, heat and salt stresses (the absolute value of Fold change $>1, p$-value $<0.05$ ), respectively. To provide more evidences for the RsbZIP genes' roles in response to different abiotic stresses, the expression of the 10 RsbZIP genes (RsbZIP005/006/010/031/059/064/076/077/092/101), which were selectively expressed at high-levels in taproot, was analyzed under $\mathrm{Cd}, \mathrm{Cr}, \mathrm{Pb}$, heat and salt stresses. As shown in Figure $6 \mathrm{~b}-\mathrm{f}$, seven of these 10 RsbZIP genes exhibited significantly differential expression in taproot under the $\mathrm{Cd}, \mathrm{Cr}, \mathrm{Pb}$, heat and salt treatments. For example, the RsbZIP010 gene exhibited down-regulated expression under three heavy metal (HM) stresses, whereas the RsbZIP005 gene was significantly up- and down-regulated under Cd and $\mathrm{Cr}$ stress, respectively (Figure $6 \mathrm{~b}-\mathrm{d}$ ), indicating that the RsbZIP010 gene might play conserved key roles in the biological process of response to three HM stresses in radish taproot. Notably, the RsbZIP031 and RsbZIP059 genes showed significant down-regulation under heat and salt stresses, respectively (Figure 6e,f). This finding indicated that these two genes may act as critical regulators in heat and salt stress response in radish taproot. However, there was no expression of RsbZIP076, RsbZIP077 and RsbZIP101 in taproot under the five abiotic stresses. To validate the expression profiles of RsbZIP genes obtained from the RNA-seq data, seven high-expressed RsbZIP (RsbZIP005/006/010/031/059/064/092) genes in taproot combined with another six RsbZIP genes were selected for real-time quantitative polymerase chain reaction (RT-qPCR) analysis. The expression patterns from RT-qPCR indicated a general agreement with those from the RNA-seq results (Figure $6 b-f$, Figure S3).
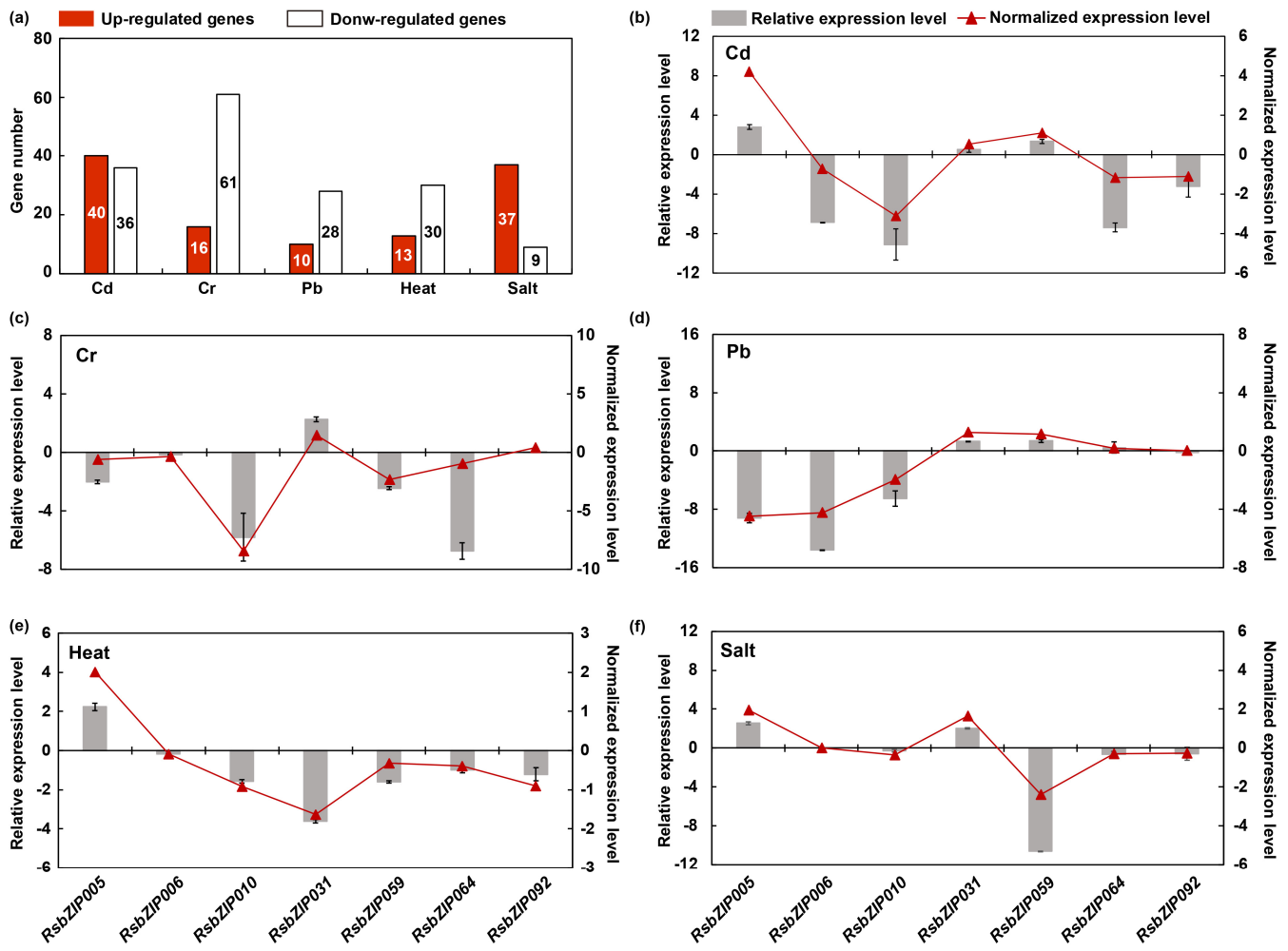

Figure 6. Expression profiles of RsbZIP genes under $\mathrm{Cd}, \mathrm{Cr}, \mathrm{Pb}$, heat and salt stresses. (a) The number of RsbZIP genes that are up- and down-regulated (the absolute value of Fold change $>1, p$-value $<0.05$ ) under $\mathrm{Cd}, \mathrm{Cr}, \mathrm{Pb}$, heat and salt stresses. (b-f) Expression profiles of seven RsbZIP genes that specifically high-expressed in taproot under $\mathrm{Cd}, \mathrm{Cr}, \mathrm{Pb}$, heat and salt stresses from RNA-seq data and RT-qPCR analysis. Red bar represents transcript abundance change calculated by the RPKM method. Gray bar with associated standard error bar indicates relative expression level determined by RT-qPCR analysis. 


\subsection{Potential Roles of RsbZIP Genes in Anthocyanin Biosynthetic Pathway}

Two Group H members of Arabidopsis bZIP family, HY5 (AtbZIP56) and HYH (AtbZIP64), can be acted as important participants in the process of anthocyanin accumulation [1,14]. In this study, seven radish bZIP genes (RsbZIP011/028/063/065/082/097/102) that were homologous to Arabidopsis HY5 and $H Y H$ were used for expression profiles in ten radish genotypes with red-skin and white-skin using RT-qPCR. The results showed that the expression levels of RsbZIP011 and RsbZIP102 were significantly up-regulated in five red-skin genotypes compared with that in five white-skin ones (Figure 7a,b, Figure S4). Furthermore, RsbZIP011 and RsbZIP102 also exhibited higher expression levels in five tissues (root skin, stem, leaf vein, septal and petal) with high anthocyanin contents than that in root flesh with low anthocyanin content (Figure 7c-e), indicating that RsbZIP011 and RsbZIP102 may participate in anthocyanin biosynthetic pathway in radish.

(a)

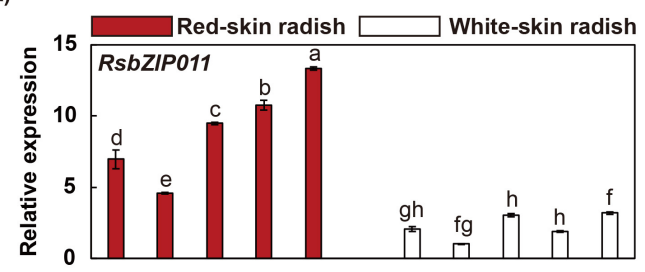

(b)

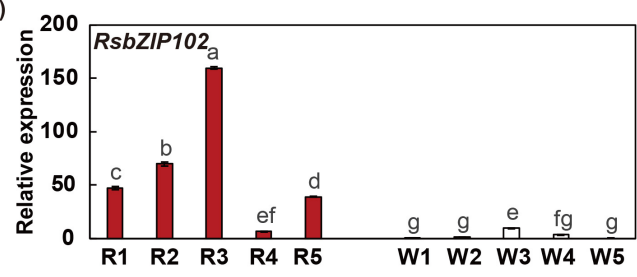

(c)

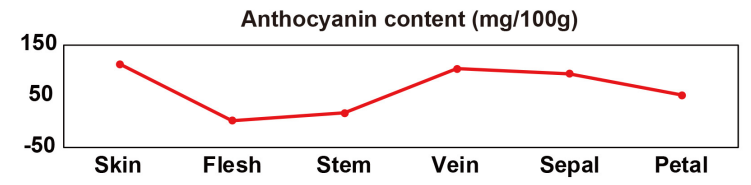

(d)

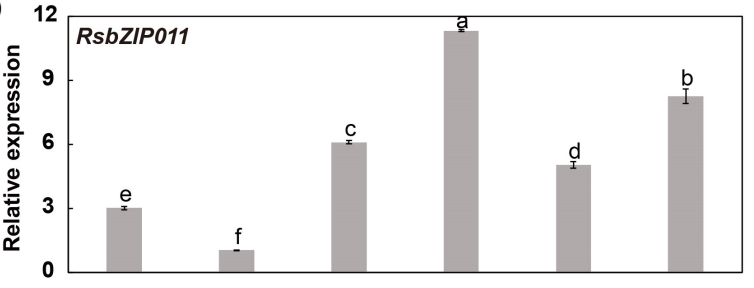

(e)

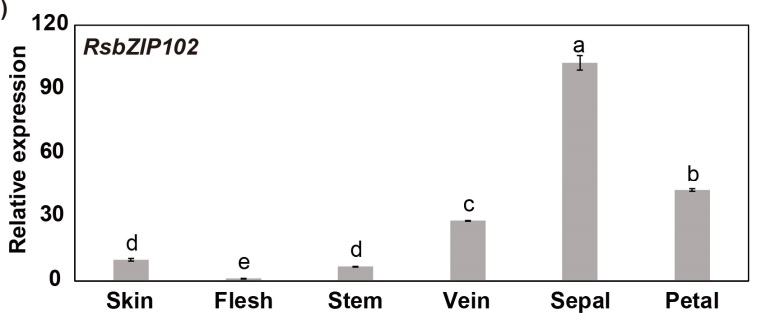

Figure 7. Expression profiles of RsbZIP genes potentially involved in anthocyanin biosynthesis pathway. Here, (a) and (b) show the expression profiles of RsbZIP011 and RsbZIP102 in five red-skin (R1, R2, R3, R4, R5) and five white-skin radish genotypes (W1, W2, W3, W4, W5). (c) Anthocyanin content in six different tissues (skin, flesh, stem. vein, sepal and petal) of radish. Moreover, (d) and (e) Expression profiles of RsbZIP011 and RsbZIP102 in six different tissues. Error bars indicate standard deviation based on three replicates. Letters represent significant differences at a 0.05 level based on Duncan's test.

\section{Discussion}

\subsection{Characterization of bZIP Gene Family Members in Radish}

The $b$ ZIP gene family, one of the largest TF families, has been extensively characterized in many plants, including 78 bZIP genes in Arabidopsis [5], 69 in tomato [7], 89 in rice [8] and 247 in rapeseed [10]. In this study, $135 b Z I P$ genes were identified from the radish genome, indicating that the $b Z I P$ gene family in radish had been expanded compared to that in Arabidopsis, rice and tomato. Phylogenetic analysis indicated that the radish bZIP family could be divided into 12 subfamilies based on cluster analysis and a comparison with Arabidopsis bZIP genes. The phylogenetic relationship of RsbZIPs was also supported by both their gene structure and conserved motif.

Gene structure analysis showed that RsbZIP genes possessed intron with number varying from 0 to 12 , and each subfamily displayed similar exon-intron organizations. In total, 44 RsbZIP genes contained lower intron number (0 or 1 intron), which is similar in soybean [4], apple [9] and rapeseed [10]. 
Consistent with the results in soybean and rapeseed, the RsbZIP genes from Groups D and G contained more than six introns, suggesting that they might contain the original RsbZIP genes in these groups $[4,10]$. In this study, exon/intron gain/loss was also observed. For instance, RsZIP050 contained 13 exons, while its paralogous RsbZIP135 had 11 exons (Figure 2), indicating a loss of two exons occurred during evolution. A similar pattern was also reported in the apple and soybean bZIP families [4,9]. These gain/losses may be the results of chromosomal rearrangements and fusions, and can potentially lead to the functional diversification of multiple gene families [24]. Additionally, two genes in a duplicated gene pair tended to be clustered into one group. The tandem duplicated pairs RsbZIP005-RsbZIP006 and RsbZIP037-RsbZIP038 were clustered into Groups A and I, respectively. The segmental pairs RsbZIP002-RsbZIP078 and RsbZIP106-RsbZIP118 were divided into Groups S and D, respectively. These duplicated genes had similar exon-intron organizations or nearly identical lengths, indicating that segmental or tandem duplication might contribute to the expansion of RsbZIP gene family [4].

Conserved motif analysis showed that all the RsbZIP proteins shared the typical bZIP domain (Motif 1) and variable Leu zipper regions (Motifs 2, 5, 8 and 10), and each subfamily harbored similar motifs. Motifs 12 and 20 shared the same domain such as the potential casein kinase II phosphorylation sites (S/TxxD/E), which were contained only in the Group A. Motifs 15 and 16 in Group G were part of a proline-rich activation domain. These findings indicated that some RsbZIP subfamily members might be conserved. In summary, the same subfamily tends to share similar exon-intron organization and motif composition, which is also reported in rapeseed [10].

\subsection{Collinearity-Orthologues of the bZIP Genes between Radish and Arabidopsis}

Comparative genomic analysis across different taxa relies on the genome structure into syntenic blocks that display conserved features across the genomes [25], which allows the transfer of functional information from a well-studied taxon (e.g., the model plant Arabidopsis) to the less-studied taxon. In this study, the synteny analysis integrating with evolutionary classification was performed to assess the relationship of $b Z I P$ genes between radish and Arabidopsis. A total of 104 radish and 63 Arabidopsis bZIP genes were identified as syntenic orthologs. Of these, nine pairs appeared to be single radish-to-Arabidopsis pairs, indicating that these genes likely present in the genome of the last common ancestor of these two species. The remaining gene combinations showed a more complex relationship, such as single radish-to-multiple Arabidopsis genes, one Arabidopsis-to-multiple radish genes and two Arabidopsis-to-multiple radish genes.

In addition, a small proportion of the bZIP genes from these two species could not be mapped into any syntenic blocks, leading into impossibility to determine whether these $b$ ZIPs share a common ancestor. This phenomenon may be attributed to two factors, which is also true for the collinearity analysis between Arabidopsis and other species such as apple [9]. Firstly, the radish and Arabidopsis genomes have undergone multiple rounds of significant chromosomal rearrangement and fusions. Secondly, selective gene loss can severely obscure the identification of chromosomal syntenies. It could be concluded that some radish $6 Z I P$ genes tend to share a common ancestor with their Arabidopsis counter parts. Moreover, AtbZIP01 and its partner AtbZIP53 control metabolic reprogramming upon salt stress in roots [26], while one of their orthologues, RsbZIP123 was also highly inducible under salt stress in this study (Figure S3). Collectively, comparative genomic analysis between radish and Arabidopsis would serve as a valuable reference for further investigation of the biological functions of RsbZIP genes.

\subsection{Roles of RsbZIP Genes in Different Tissues and Abiotic Stress Responses}

Increasing evidences indicate that the $b Z I P$ genes can act as key components under a wide range of abiotic stresses including extreme temperature and high salinity [11,12]. The transcriptome data showed that all 135 RsbZIP genes exhibited diverse expression profiles in different tissues. Nearly a third (41/135) of RsbZIP genes had high transcriptional abundance in all tissues, indicating that they may play a broad role in radish growth and development. Ten RsbZIPs were specific high-expression 
genes in radish taproot, suggesting their potential functions during the development of radish taproot. Expression profiling based on RNA-seq data and RT-qPCR analysis further showed that seven of these 10 RsbZIP genes exhibited significantly differential expression in taproot under the $\mathrm{Cd}, \mathrm{Cr}, \mathrm{Pb}$, heat and salt treatments. In particular, RsbZIP010 exhibited down-regulated expression under Cd, $\mathrm{Cr}$ and Pb stresses, whereas RsbZIP031 and RsbZIP059 showed significant down-regulation under heat and salt stresses, respectively. However, RsbZIP031 was up-regulated under salt and three $\mathrm{HM}$ stresses, showing its involvement in response to salt stress and HM detoxification. Moreover, RsbZIP059 was up-regulated under Pb and Cd stresses. These RsbZIP genes might play conserved roles in the biological process of five abiotic stress responses in radish taproot. This finding was also supported by gene ontology (GO) and cis-element analyses of RsbZIPs (Figure S5, Tables S4 and S5). GO annotation showed that 43 RsbZIP proteins were classified into response to stimulus, and stress-related cis-elements including the ABRE and ERE were found in the promoter regions of most RsbZIP genes, which is consistent with the results observed in other plants including grapevine and soybean $[4,6]$. These results indicated that the bZIP gene might play vital roles in abiotic stress response in radish taproot.

\subsection{Potential Function of bZIP Genes in Regulation of Anthocyanin Biosynthesis}

Anthocyanins are a valuable source of phytochemicals and offer benefits for human health $[27,28]$. Anthocyanins are known to be synthesized as a protective compound when the plants are exposed to various stressful events, such as low temperature, drought, ultraviolet radiation, high-intensity light and nutrient deficiency $[15,29]$. In Arabidopsis, tomato and apple, HY5 is a positive regulator during anthocyanin accumulation through direct binding to the G-box (CACGTG) element or ACGT-containing element (ACE) of anthocyanin-related gene promoters [13-15]. Here, RT-qPCR analysis further showed that the expression levels of RsbZIP011 and RsbZIP102 from Group H were associated with anthocyanin accumulation in radish. The G-box or ACE-box elements were further found in the promoters of 39 genes involved in anthocyanin biosynthesis pathway, such as Rs065380 (Chalcone synthase), Rs063340 (Chalcone flavanone isomerase), Rs341050 (Flavanone-3-hydroxylase), Rs119880 (Anthocyanidin synthase), Rs442410 (UDP-glucose flavonoid 3-o-glucosyltransferase), Rs358990 (Phenylalanine ammonia-lyase) and Rs131400 (Cinnamate-4-hydroxylase) (Table S6). It is reasonable to hypothesize that some anthocyanin-related genes may be regulated by the Arabidopsis HY5 homolog genes, such as RsbZIP011 and RsbZIP102 in radish.

Additionally, the promoters of two potentially anthocyanin-related genes (RsbZIP011 and RsbZIP102) contained several similar cis-acting elements involved in ABRE, MBS and G-box element (Table S7), indicating that they may be involved in ABA-dependent or independent stress tolerance, and could be regulated by MYB or other bZIP TFs. Thus, they may be related not only to anthocyanin biosynthesis but also to other stress responses. The Arabidopsis HY5 was found to have multifaceted roles in plant growth and development [14]. Moreover, the biotic or abiotic stressors (such as cold or heat stress) could significantly induce anthocyanin accumulation [30,31]. Therefore, it could be inferred that bZIP members might be served as a linkage between stressor and anthocyanin biosynthesis in radish.

In summary, this study describes the systematic genome- and transcriptome-wide characterization of $b$ ZIP gene family in radish. The phylogenetic classification, gene structures and conserved motifs of $135 \mathrm{bZIP}$ members were investigated. Transcriptomic and RT-qPCR analysis revealed diverse expression profiles of RsbZIP genes in taproot. Notably, the RsbZIP010 gene exhibited down-regulated expression under $\mathrm{Cd}, \mathrm{Cr}$ and $\mathrm{Pb}$ stresses, indicating that it might play conserved key roles in the biological process of response to three HM stresses in radish taproot. Except the heat stress, the RsbZIP031 gene was up-regulated under salt and three HM stresses, suggesting it might act as an important regulator in the regulation of salt and HM stress responses. Moreover, it was evidenced that RsbZIP011 and RsbZIP102 genes were important potential participants in anthocyanin biosynthesis pathway in radish. Further functional characterization of these RsbZIP genes would be useful for systematical elucidation of the 
molecular mechanism underlying the abiotic stress response and anthocyanin biosynthesis in radish plants. The outcomes of this study can not only facilitate understanding of the complexity of the bZIP gene family, but also provide novel insight into their crucial roles in abiotic stress responses and anthocyanin biosynthesis in radish.

\section{Materials and Methods}

\subsection{Identification and Phylogenetic Analysis of the bZIP Gene Family in Radish}

The protein annotation file was retrieved from Radish Genome Database (RGD, http://radishgenome.org/) [32]. The candidate bZIP genes that included at least one bZIP domain (PF00170, PF07716 or PF03131) were identified using the Pfam protein family database (http://pfam.sanger.ac.uk/) and HMMER 3.0 software (http://hmmer.janelia.org/) [33]. Subsequently, InterPro (http://www.ebi.ac.uk/ interpro/) [34] and SMART (http://smart.embl-heidelberg.de/) [35,36] programs were performed to confirm the integrity of the bZIP domain.

All of the Arabidopsis bZIP protein sequences [1] were obtained from TAIR database (https: //www.arabidopsis.org/). The aa sequences of radish and Arabidopsis bZIPs (Table S1) were imported into MEGA $X$ and multiple sequence alignments were performed using MUSCLE [37]. Thereafter, the alignment file was used to construct $\mathrm{NJ}$ phylogenetic tree with following parameters: p-distance, pairwise deletion and bootstrap (1000 replicates) [37,38]. The NJ tree was visualized using Evolview v2 (http://www.evolgenius.info/evolview/) [39]. The phylogenetic tree of all radish bZIP family members was also generated by MEGA $X$ with the same parameters.

\subsection{Synteny Analysis and Chromosomal Localization}

BLASTP search was executed against all potential anchors $\left(E<1 \times 10^{-5}\right)$ within the radish genome and between the radish and Arabidopsis genomes. Collinear blocks were evaluated using MCScanX software, and alignments with $\leq 1 \times 10^{-10}$ were considered as significant matches [40]. Chromosomal position from RGD and duplications (segmental and tandem) of the RsbZIP genes were displayed by TBtools software (https://github.com/CJ-Chen/TBtools).

\subsection{Analyses of Gene Structure, Conserved Motif, Promoter and GO Annotation}

The exon-intron organizations for RsbZIP genes were illustrated with Gene Structure Display Server (http://gsds.cbi.pku.edu.cn/) [41]. Identification of conserved motifs was performed by the MEME program (http://meme.nbcr.net/meme/cgi-bin/meme.cgi) [42]. GO annotation of RsbZIP protein sequences was performed using Blast2GO (http://www.blast2go.com). The upstream $1.5 \mathrm{~kb}$ genomic sequences of radish $b Z I P$ genes were submitted to the PlantCARE database (http://bioinformatics.psb. ugent.be/webtools/plantcare/html/) to identify the putative cis-elements [43].

\subsection{Transcriptome-Based Expression Profiling of RsbZIP Genes}

The published transcriptome data [18] were used for expression pattern analysis of global RsbZIPs. The expression level for each RsbZIP gene in leaf and taproot (cortical, cambium, xylem and root tip) at 60 days after germination (DAG) was calculated using the RPKM. Heatmap of RsbZIP gene expression profile was generated by TBtools software (https://github.com/CJ-Chen/TBtools). Expression patterns of RsbZIP genes under $\mathrm{Cd}, \mathrm{Cr}, \mathrm{Pb}$, heat and salt stresses were extracted from transcriptome data of radish taproot [19-23].

\subsection{Plant Materials and Treatments}

The radish seeds of 'NAU-RG' were sown in the pots containing mixture of isovolumetric soil and substrate, and grown under a 14 -h photoperiod at approximately $25^{\circ} \mathrm{C} / 16^{\circ} \mathrm{C}$ (day/night) in the growth chamber. Twenty-day-old seedlings were subjected to different stress conditions. The $\mathrm{Cd}, \mathrm{Cr}$ and $\mathrm{Pb}$ treatments were carried out by soaking the $200 \mathrm{mg} / \mathrm{L} \mathrm{CdCl} 2 \cdot 2.5 \mathrm{H}_{2} \mathrm{O}$ for $12 \mathrm{~h}, 600 \mathrm{mg} / \mathrm{L} \mathrm{K}_{2} \mathrm{Cr}_{2} \mathrm{O}_{7}$ for 
$72 \mathrm{~h}$ and $1000 \mathrm{mg} / \mathrm{L} \mathrm{Pb}\left(\mathrm{NO}_{3}\right)_{2}$ for $72 \mathrm{~h}$ [19-21]. Heat stress was simulated by $40{ }^{\circ} \mathrm{C}$ for $24 \mathrm{~h}$ in growth chamber [22] and high-salinity stress was imposed by irrigating with $200 \mathrm{mM} \mathrm{NaCl}$ for $48 \mathrm{~h}$ [23]. After different treatments, the treated roots in each stress were harvested separately for total RNA isolation.

To characterize the potential function of radish $b Z I P$ genes in anthocyanin biosynthesis, five red-skin (R1, R2, R3, R4, R5) and five white-skin (W1, W2, W3, W4, W5) genotypes were selected and grown in the growth chamber. The root skins of all 10 radish genotypes were harvested at 40 DAG. Additionally, the seeds of one red-skin genotype 'R1' were vernalized at $4{ }^{\circ} \mathrm{C}$ for 10 days and grown until flowering and podding. Six tissue (root skin, flesh, stem, leaf vein, sepal and petal) samples were separately collected, which were frozen immediately in liquid nitrogen and stored at $-80^{\circ} \mathrm{C}$ for further use. For each treatment or tissue, three independent replicates were sampled.

\subsection{Determination of Anthocyanin Content}

The anthocyanins from all above-mentioned samples were extracted in accordance with the method described by Guo et al. [44]. In brief, the supernatant was filtered through $0.22-\mu \mathrm{m}$ filters for anthocyanin content analysis. The $\mathrm{pH}$ differential spectrophotometry method was used to determine total anthocyanin contents in different tissues [44]. Measurements of all samples were replicated three times.

\subsection{RT-qPCR Analysis}

Total RNA extraction was performed with RNAprep Pure Plant Kit (Tiangen, Beijing, China) for RT-qPCR analysis. First-strand cDNA was synthesized using PrimeScript ${ }^{\mathrm{TM}}$ RT reagent kit with gDNA eraser (Takara, Dalian, China). Gene expression profiles were generated in triplicate employing a RT-qPCR approach on a LightCycler ${ }^{\circledR} 480$ System (Roche, Mannheim, Germany) according to the manufacturer's instructions. The specific primers were listed in Table S8. The relative expression level was normalized to the RsActin gene and calculated by the $2^{-\Delta \Delta \mathrm{C} T}$ method [45].

Supplementary Materials: Supplementary materials can be found at http://www.mdpi.com/1422-0067/20/24/ 6334/s1. Figure S1: The variations of leucine zipper region for RsbZIP proteins, Figure S2: Venn diagrams of overlapping RsbZIPs that are expressed in leaf, cortical, cambium, xylem and root tip when RPKM are greater than 2, 4, 6 and 8, respectively, Figure S3: RT-qPCR validation of selected six RsbZIPs under Cd, Cr, Pb, heat and salt treatments in radish taproot, Figure S4: Expression profiles of several RsbZIP genes from Group H (RsbZIP028, 063, 065, 082 and 097) in five red-skin (R1, R2, R3, R4, R5) and five white-skin radish genotypes (W1, W2, W3, W4, W5), Figure S5: GO annotation of RsbZIP proteins, Table S1: Information of bZIP genes from radish and Arabidopsis, Table S2: Segmental duplicated $b$ ZIP gene pairs within radish genome, Table S3: Synteny blocks of bZIP genes between radish and Arabidopsis genomes, Table S4: GO annotation details of RsbZIP proteins, Table S5: Cis-element analysis of RsbZIP promoters, Table S6: Promoter sequences of genes involved in anthocyanin biosynthesis, Table S7: Cis-regulation elements of RsbZIP genes potentially related to anthocyanin biosynthesis, Table S8: List of primers used in RT-qPCR analysis.

Author Contributions: Conceptualization, L.F., L.X. and L.L.; Writing-original draft preparation, L.F. and L.X.; Writing-review and editing, L.X., Y.W. and L.L.; Visualization, L.F. and M.T.; Validation, L.F.; Project administration, L.L.

Funding: This research was funded by grants from the National Key Technology R\&D Program of China (2017YFD0101806/03; 2018YFD1000800), the Jiangsu Agricultural S\&T Innovation Fund [CX(19)3032, CX(18)3067], Jiangsu Agricultural Industry Technology System (JATS[2019]428) and the "333 project" of Jiangsu Province (BRA2018063).

Conflicts of Interest: The authors declare no conflict of interest. 


\section{Abbreviations}

$\begin{array}{ll}\text { Amino acids } & \text { aa } \\ \text { bZIP } & \text { Basic leucine zipper } \\ \mathrm{Cd} & \text { Cadmium } \\ \mathrm{Cr} & \text { Chromium } \\ \mathrm{Chr}(\mathrm{s}) & \text { Chromosome(s) } \\ \mathrm{DAG} & \text { Days after germination } \\ \mathrm{GO} & \text { Gene ontology } \\ \mathrm{HM} & \text { Heavy metal } \\ \mathrm{HY} 5 & \text { Elongated hypocotyl } 5 \\ \mathrm{HYH} & \text { HY5 homolog } \\ \mathrm{Pb} & \text { Lead } \\ \text { Leu } & \text { Leucine } \\ \mathrm{NJ} & \text { Neighbor-Joining } \\ \text { RGD } & \text { Radish Genome Database } \\ \text { RPKM } & \text { Reads per kilobase per million reads } \\ \text { RT-qPCR } & \text { Real-Time Quantitative Polymerase Chain Reaction }\end{array}$

\section{References}

1. Dröge-Laser, W.; Snoek, B.L.; Snel, B.; Weiste, C. The Arabidopsis bZIP transcription factor family-An update. Curr. Opin. Plant Biol. 2018, 45, 36-49. [CrossRef] [PubMed]

2. Wang, Z.; Cheng, K.; Wan, L.; Yan, L.; Jiang, H.; Liu, S.; Lei, Y.; Liao, B. Genome-wide analysis of the basic leucine zipper (bZIP) transcription factor gene family in six legume genomes. BMC Genom. 2015, 16, 1053. [CrossRef] [PubMed]

3. Unel, N.M.; Cetin, F.; Karaca, Y.; Celik, A.Y.; Baloglu, M.C. Comparative identification, characterization, and expression analysis of $b$ ZIP gene family members in watermelon and melon genomes. Plant Growth Regul. 2019, 87, 227-243. [CrossRef]

4. Zhang, M.; Liu, Y.; Shi, H.; Guo, M.; Chai, M.; He, Q.; Yan, M.; Cao, D.; Zhao, L.; Cai, H.; et al. Evolutionary and expression analyses of soybean basic Leucine zipper transcription factor family. BMC Genom. 2018, 19, 159. [CrossRef] [PubMed]

5. Jakoby, M.; Weisshaar, B.; Dröge-Laser, W.; Vicente-Carbajosa, J.; Tiedemann, J.; Kroj, T.; Parcy, F. bZIP transcription factors in Arabidopsis. Trends Plant Sci. 2002, 7, 106-111. [CrossRef]

6. Liu, J.; Chen, N.; Chen, F.; Bin, C.; Santo, S.D.; Tornielli, G.B.; Pezzotti, M.; Cheng, Z.M. Genome-wide analysis and expression profile of the bZIP transcription factor gene family in grapevine (Vitis vinifera). BMC Genom. 2014, 15, 281. [CrossRef]

7. Li, D.; Fu, F.; Zhang, H.; Song, F. Genome-wide systematic characterization of the bZIP transcriptional factor family in tomato (Solanum lycopersicum L.). BMC Genom. 2015, 16, 771. [CrossRef]

8. Nijhawan, A.; Jain, M.; Tyagi, A.K.; Khurana, J.P. Genomic survey and gene expression analysis of the basic leucine zipper transcription factor family in rice. Plant Physiol. 2008, 146, 333-350. [CrossRef]

9. Zhao, J.; Guo, R.; Guo, C.; Hou, H.; Wang, X.; Gao, H. Evolutionary and expression analyses of the apple basic leucine zipper transcription factor family. Front. Plant Sci. 2016, 7, 376. [CrossRef]

10. Zhou, Y.; Xu, D.; Jia, L.; Huang, X.; Ma, G.; Wang, S.; Zhu, M.; Zhang, A.; Guan, M.; Lu, K.; et al. Genome-wide identification and structural analysis of bZIP transcription factor genes in Brassica napus. Genes 2017, 8, 288. [CrossRef]

11. Zhang, S.S.; Yang, H.; Ding, L.; Song, Z.T.; Ma, H.; Chang, F.; Liu, J.X. Tissue-specific transcriptomics reveals an important role of the unfolded protein response in maintaining fertility upon heat stress in Arabidopsis. Plant Cell 2017, 29, 1007-1023. [CrossRef] [PubMed]

12. Zhu, M.; Meng, X.; Cai, J.; Li, G.; Dong, T.; Li, Z. Basic leucine zipper transcription factor SlbZIP1 mediates salt and drought stress tolerance in tomato. BMC Plant Biol. 2018, 18, 83. [CrossRef] [PubMed]

13. An, J.P.; Qu, F.J.; Yao, J.F.; Wang, X.N.; You, C.X.; Wang, X.F.; Hao, Y.J. The bZIP transcription factor MdHY5 regulates anthocyanin accumulation and nitrate assimilation in apple. Hortic. Res. 2017, 4, 17023. [CrossRef] [PubMed] 
14. Gangappa, S.N.; Botto, J.F. The multifaceted roles of HY5 in plant growth and development. Mol. Plant 2016, 9, 1353-1365. [CrossRef] [PubMed]

15. Liu, C.C.; Chi, C.; Jin, L.J.; Zhu, J.; Yu, J.Q.; Zhou, Y.H. The bZip transcription factor HY5 mediates CRY1a-induced anthocyanin biosynthesis in tomato. Plant Cell Environ. 2018, 41, 1762-1775. [CrossRef] [PubMed]

16. Kitashiba, H.; Li, F.; Hirakawa, H.; Kawanabe, T.; Zou, Z.; Hasegawa, Y.; Tonosaki, K.; Shirasawa, S.; Fukushima, A.; Yokoi, S.; et al. Draft sequences of the radish (Raphanus sativus L.) genome. DNA Res. 2014, 21, 481-490. [CrossRef] [PubMed]

17. Sun, Y.; Qiu, Y.; Duan, M.; Wang, J.; Zhang, X.; Wang, H.; Song, J.; Li, X. Identification of anthocyanin biosynthesis related microRNAs in a distinctive Chinese radish (Raphanus sativus L.) by high-throughput sequencing. Mol. Genet. Genom. 2017, 292, 215-229. [CrossRef]

18. Mitsui, Y.; Shimomura, M.; Komatsu, K.; Namiki, N.; Shibata-Hatta, M.; Imai, M.; Yuichi, K.; Mukai, Y.; Kanamori, H.; Kurita, K.; et al. The radish genome and comprehensive gene expression profile of tuberous root formation and development. Sci. Rep. 2015, 5, 10835. [CrossRef]

19. Xu, L.; Wang, Y.; Liu, W.; Wang, J.; Zhu, X.; Zhang, K.; Yu, R.; Wang, R.; Xie, Y.; Zhang, W.; et al. De novo sequencing of root transcriptome reveals complex cadmium-responsive regulatory networks in radish (Raphanus sativus L.). Plant Sci. 2015, 236, 313-323. [CrossRef]

20. Xie, Y.; Ye, S.; Wang, Y.; Xu, L.; Zhu, X.; Yang, J.; Feng, H.; Yu, R.; Karanja, B.; Gong, Y.; et al. Transcriptome-based gene profiling provides novel insights into the characteristics of radish root response to Cr stress with next-generation sequencing. Front. Plant Sci. 2015, 6, 202. [CrossRef]

21. Wang, Y.; Xu, L.; Chen, Y.; Shen, H.; Gong, Y.; Limera, C.; Liu, L. Transcriptome profiling of radish (Raphanus sativus $\mathrm{L}$.) root and identification of genes involved in response to Lead ( $\mathrm{Pb}$ ) stress with next generation sequencing. PLoS ONE 2013, 8, e66539. [CrossRef] [PubMed]

22. Wang, R.; Mei, Y.; Xu, L.; Zhu, X.; Wang, Y.; Guo, J.; Liu, L. Genome-wide characterization of differentially expressed genes provides insights into regulatory network of heat stress response in radish (Raphanus sativus L.). Funct. Integr. Genom. 2018, 18, 225-239. [CrossRef]

23. Sun, X.; Xu, L.; Wang, Y.; Luo, X.; Zhu, X.; Kinuthia, K.B.; Nie, S.; Feng, H.; Li, C.; Liu, L. Transcriptome-based gene expression profiling identifies differentially expressed genes critical for salt stress response in radish (Raphanus sativus L.). Plant Cell Rep. 2016, 35, 329-346. [CrossRef] [PubMed]

24. Xu, G.; Guo, C.; Shan, H.; Kong, H. Divergence of duplicate genes in exon-intron structure. Proc. Natl. Acad. Sci. USA 2012, 109, 1187-1192. [CrossRef] [PubMed]

25. Ghiurcuta, C.G.; Moret, B.M. Evaluating synteny for improved comparative studies. Bioinformatics 2014, 30, i9-i18. [CrossRef]

26. Hartmann, L.; Pedrotti, L.; Weiste, C.; Fekete, A.; Schierstaedt, J.; Göttler, J.; Kempa, S.; Krischke, M.; Dietrich, K.; Mueller, M.J.; et al. Crosstalk between two bZIP signaling pathways orchestrates salt-induced metabolic reprogramming in Arabidopsis roots. Plant Cell 2015, 27, 2244-2260. [CrossRef]

27. Lim, S.H.; Song, J.H.; Kim, D.H.; Kim, J.K.; Lee, J.Y.; Kim, Y.M.; Ha, S.H. Activation of anthocyanin biosynthesis by expression of the radish R2R3-MYB transcription factor gene RsMYB1. Plant Cell Rep. 2016, 35, 641-653. [CrossRef]

28. Naing, A.H.; Kim, C.K. Roles of R2R3-MYB transcription factors in transcriptional regulation of anthocyanin biosynthesis in horticultural plants. Plant Mol. Biol. 2018, 98, 1-18. [CrossRef]

29. Costantini, L.; Malacarne, G.; Lorenzi, S.; Troggio, M.; Mattivi, F.; Moser, C.; Grando, M.S. New candidate genes for the fine regulation of the colour of grapes. J. Exp. Bot. 2015, 66, 4427-4440. [CrossRef]

30. Ilk, N.; Ding, J.; Ihnatowicz, A.; Koornneef, M.; Matthieu, R. Natural variation for anthocyanin accumulation under high-light and low-temperature stress is attributable to the ENHANCER OF AG-4 2 (HUA2) locus in combination with PRODUCTION OF ANTHOCYANIN PIGMENT1 (PAP1) and PAP2. New Phytol. 2015, 206, 422-435. [CrossRef]

31. Rienth, M.; Torregrosa, L.; Luchaire, N.; Chatbanyong, R.; Lecourieux, D.; Kelly, M.T.; Romieu, C. Day and night heat stress trigger different transcriptomic responses in green and ripening grapevine (Vitis vinifera) fruit. BMC Plant Biol. 2014, 14, 108. [CrossRef] [PubMed]

32. Jeong, Y.M.; Kim, N.; Ahn, B.O.; Oh, M.; Chung, W.H.; Chung, H.; Jeong, S.; Lim, K.B.; Hwang, Y.J.; Kim, G.B.; et al. Elucidating the triplicated ancestral genome structure of radish based on chromosome-level comparison with the Brassica genomes. Theor. Appl. Genet. 2016, 129, 1357-1372. [CrossRef] [PubMed] 
33. Finn, R.D.; Coggill, P.; Eberhardt, R.Y.; Eddy, S.R.; Mistry, J.; Mitchell, A.L.; Potter, S.C.; Punta, M.; Qureshi, M.; Sangrador-Vegas, A.; et al. The Pfam protein families database: Towards a more sustainable future. Nucleic Acids Res. 2016, 44, D279-D285. [CrossRef] [PubMed]

34. Finn, R.D.; Attwood, T.K.; Babbitt, P.C.; Bateman, A.; Bork, P.; Bridge, A.J.; Chang, H.Y.; Dosztányi, Z.; El-Gebali, S.; Fraser, M.; et al. InterPro in 2017-beyond protein family and domain annotations. Nucleic Acids Res. 2017, 45, D190-D199. [CrossRef]

35. Han, Z.; Liu, Y.; Deng, X.; Liu, D.; Liu, Y.; Hu, Y.; Yan, Y. Genome-wide identification and expression analysis of expansin gene family in common wheat (Triticum aestivum L.). BMC Genom. 2019, 20, 101. [CrossRef]

36. Letunic, I.; Doerks, T.; Bork, P. Smart: Recent updates, new developments and status in 2015. Nucleic Acids Res. 2015, 43, D257-D260. [CrossRef]

37. Kumar, S.; Stecher, G.; Li, M.; Knyaz, C.; Tamura, K. MEGA X: Molecular evolutionary genetics analysis across computing platforms. Mol. Biol. Evol. 2018, 35, 1547-1549. [CrossRef]

38. Sanjari, S.; Shirzadian-Khorramabad, R.; Shobbar, Z.S.; Shahbazi, M. Systematic analysis of NAC transcription factors' gene family and identification of post-flowering drought stress responsive members in sorghum. Plant Cell Rep. 2019, 38, 361-376. [CrossRef]

39. He, Z.; Zhang, H.; Gao, S.; Lercher, M.J.; Chen, W.H.; Hu, S. Evolview v2: An online visualization and management tool for customized and annotated phylogenetic trees. Nucleic Acids Res. 2016, 44, W236-W241. [CrossRef]

40. Wang, Y.; Tang, H.; Debarry, J.D.; Tan, X.; Li, J.; Wang, X.; Lee, T.; Jin, H.; Marler, B.; Guo, H.; et al. MCScanX: A toolkit for detection and evolutionary analysis of gene synteny and collinearity. Nucleic Acids Res. 2012, 40, e49. [CrossRef]

41. Hu, B.; Jin, J.; Guo, A.Y.; Zhang, H.; Luo, J.; Gao, G. GSDS 2.0: An upgraded gene feature visualization server. Bioinformatics 2015, 31, 1296-1297. [CrossRef] [PubMed]

42. Bailey, T.L.; Johnson, J.; Grant, C.E.; Noble, W.S. The MEME suite. Nucleic Acids Res. 2015, 43, W39-W49. [CrossRef] [PubMed]

43. Wang, P.; Su, L.; Gao, H.; Jiang, X.; Wu, X.; Li, Y.; Zhang, Q.; Wang, Y.; Ren, F. Genome-wide characterization of $b H L H$ genes in grape and analysis of their potential relevance to abiotic stress tolerance and secondary metabolite biosynthesis. Front. Plant Sci. 2018, 9, 64. [CrossRef] [PubMed]

44. Guo, N.; Wu, J.; Zheng, S.; Cheng, F.; Liu, B.; Liang, J.; Cui, Y.; Wang, X. Anthocyanin profile characterization and quantitative trait locus mapping in zicaitai (Brassica rapa L. ssp. Chinensis var. purpurea). Mol. Breed. 2015, 35, 113. [CrossRef]

45. Xu, Y.; Zhu, X.; Gong, Y.; Xu, L.; Wang, Y.; Liu, L. Evaluation of reference genes for gene expression studies in radish (Raphanus sativus L.) using quantitative real-time PCR. Biochem. Biophys. Res. Commun. 2012, 424, 398-403. [CrossRef]

(C) 2019 by the authors. Licensee MDPI, Basel, Switzerland. This article is an open access article distributed under the terms and conditions of the Creative Commons Attribution (CC BY) license (http://creativecommons.org/licenses/by/4.0/). 